\title{
Clinico-biochemical responses of dogs to experimental infection with Babesia canis
}

\author{
M. Konto, A. A. Biu, M. I. Ahmed, A. W. Mbaya and J. Luka \\ Department of Veterinary Microbiology and Parasitology, \\ Faculty of Veterinary Medicine, University of Maiduguri, PMB 1069 Maiduguri, Borno State, Nigeria. \\ Corresponding author: M. Konto, email: kontomohammed@yahoo.com \\ Received: 22-11-2013, Revised: 30-01-2014, Accepted: 04-02-2014, Published online: 04-03-2014
}

doi: 10.14202 /vetworld.2014.113-118

How to cite this article: Konto M, Biu AA, Ahmed MI, Mbaya AW and Luka J (2014) Clinico-biochemical responses of dogs to experimental infection with Babesia canis, Veterinary World 7(3): 113-118.

\begin{abstract}
Aim: A study on the clinical and biochemical parameters of Nigerian dogs experimentally infected with Babesia canis was conducted.

Materials and Methods: A total of ten naive dogs of both sex and aged between 6 months to 1 year, were used for the study. They were divided into two groups of five each- A (control) and B (infected). Dogs in group B were infected with 1ml of Babesia canis positive infectious inoculum, while those in group A were left as uninfected control. Following infection, clinical and biochemical responses were analyzed in group B and compared with those in group A.

Results: Clinical signs were observed on the infected dogs 2 days post infection, which included fever (100\%), increase in pulse $(80 \%)$, tachycardia $(60 \%)$ and inappetence $(100 \%)$; followed by anorexia $(40 \%)$ and lethargy $(100 \%)$ on day 3 ; on the fourth day, pallor of the mucous membrane of the mouth and eye (100\%) and emaciation (100\%); on day five, muscle tremor $(20 \%)$ and respiratory distress (20\%); on day six, nervousness (20\%), drooling salivation (20\%) and haemoglobinuria (80); on day seven, mucoid ocular discharge $(40 \%)$; followed by the death of one dog on day 8 post infection. Other clinical signs recorded between days 1-14 post infection were ascites, edematous swelling of the whole body and hair erection. The biochemical changes showed that there was a significant $(\mathrm{p}<0.05)$ rise in alkaline phosphatase (ALP) values in infected dogs $(58.50 \pm 1.4)$ compared with the control group $(51.67 \pm 1.6)$. Also, there was a significant rise $(\mathrm{p}<0.05)$ in the alanine amino transferase (ALT) values of infected group $(15.70 \pm 1.8)$ compared with the control values $(8.27 \pm 2.0)$. However, the mean values for creatinine of infected group $(78.10 \pm 1.2)$ was significantly lower $(\mathrm{p}<0.05)$ than that of the control $(91.73 \pm 1.3)$. Similarly, the glucose levels for infected group $(3.80 \pm 2.3)$ were significantly $(\mathrm{p}<0.05)$ lower than that of control $(5.35 \pm 2.1)$.
\end{abstract}

Conclusion: It can be concluded that the disease runs an acute as well as a chronic course in the study area and that Diminazene aceturate at a dose rate of $0.3 \mathrm{mg} / \mathrm{kg}$ body proved effective against parasitaemia within two weeks post chemotherapy.

Keywords: Babesia canis, biochemical, clinical, dogs, experimental infection, West Africa.

\section{I ntroduction}

Canine babesiosis caused by different Babesia species is a protozoan tick-borne disease with worldwide distribution and of global significance. Historically, Babesia infection in dogs was identified based on morphologic appearance in the erythrocyte, until recently revolutionized by the introduction of newer techniques like serological $[1,2]$ and molecular techniques like polymerase chain reaction [1-3]. Morphological analysis has demonstrated three distinct sub species of Babesia canis viz., Babesia canis rossi, Babesia canis canis and Babesia canis vogeli [4]. The three sub species are morphologically indistinguishable and demonstrate tremendous variations in clinical signs, geographical distribution and vector specificity $[5,6]$.

The parasite has been reported to be transmitted by an array of well-described Ixodid tick vectors from different geographical locations. Depending on the

Copyright: The authors. This article is an open access article licensed under the terms of the Creative Commons Attribution License (http://creativecommons.org/licenses/by/2.0) which permits unrestricted use, distribution and reproduction in any medium, provided the work is properly cited. species of Babesia parasite present in an area, the common tick vectors reported to serve in transmission of canine babesiosis include Rhipicephalus species, Haemaphysalis species and Dermacentor species [7]. A non-vectored transmission through blood exchange during fighting and biting has also been reported, as seen with some aggressive breeds of dogs like the Pit Bull terriers $[8,9]$. Other route of transmission is by transplacental route, as reported in puppies as young as 3 days old $[8,10]$.

Most of the abnormalities seen in Babesia canis infection are related both to the severity of the disease and the degree of hypoxia. The severity ranges from a mild asymptomatic infection to a severe hyperacute shock-related haemolytic crisis [11] with generalized organ failure resulting to death within few days of infection. The disease is generally characterized with regenerative haemolytic anaemia [12] and/or thrombocytopenia [13,14], and usually accompanied with inappetence to complete anorexia, fever, increase in pulse and respiratory rates, pallor of the mucous membranes of the mouth and eyelids, lethargy, splenomegaly, water-hammer pulse, icterus and pigmenturia, 
Table-1. Clinical signs associated with Babesia canis infected dogs $(n=10)$

\begin{tabular}{lcc}
\hline Clinical signs & No. (\%) of dog(s) showing sign & Days post infection \\
\hline Fever & $5(100)$ & 2 days \\
Increased pulse rate & $4(80)$ & 2 days \\
Tachycardia & $3(60)$ & 2 days \\
Inappetence & $5(100)$ & 2 days \\
Anorexia & $2(40)$ & 3 days \\
Lethargy & $5(100)$ & 3 days \\
Pallor of mucous membranes & $5(100)$ & 4 days \\
Emaciation & $5(100)$ & 4 days \\
Muscle tremor & $1(20)$ & 5 days \\
Respiratory distress & $1(20)$ & 5 days \\
Nervousness & $1(20)$ & 6 days \\
Drooling salivation & $1(20)$ & 6 days \\
Haemoglobinuria & $4(80)$ & 6 days \\
Mucoid ocular discharge & $2(40)$ & 7 days \\
Death & $1(20)$ & 8 days \\
Ascites & $2(40)$ & 10 days \\
Edematous body swelling & $1(20)$ & 12 days \\
Hair erection & $1(20)$ & 13 days \\
\hline
\end{tabular}

depending on the specie of parasite. Clinico-pathologic abnormalities reported before include hypoglycemia, acid-base disturbance, azoturia and alterations in the levels of the liver and kidney enzymes and the acute phase protein $[15,16]$. Common biochemical findings are increase in the serum activity of aspertate aminotransferase (AST) and alanine aminotransferase (ALT), hyperbilirubinaemia, hypoalbuminaemia, electrolyte and acid-base abnormalities (mostly hypokalemia, hypercloremia and metabolic acidosis) [17-20].This study was designed to determine the clinico-biochemical changes associated with canine babesiosis under experimental condition using African (Nigeria) dogs as models.

\section{Materials and Methods}

Ethical consideration: All procedures were performed with the approval of the Institutional Animal Care and Use Committee of the University of Maiduguri, Nigeria.

A total of ten naive dogs (Mongrels) of age 6 month to 1 year and of both sex were used for the experiment, following physical and clinical examination to ascertain that the dogs are clinically free from Babesia infection before experimental infection with Babesia canis. The experimental dogs were fed daily with standard dog feed and a potable drinking water ad libitum.

Preparation of infection inoculum: A puppy of less than six months of age was splenectomised following physical and clinical examination to ascertain that the dog is free from any haemoparasites. Thereafter, screened B. canis positive blood from an infected dog was used to inoculate the splenectomised puppy. Heparinized blood samples were obtained daily for the determination of PCV and parasitaemia. During patent parasitaemia, peripheral blood was collected in citrate solution and used to prepare the challenge inoculum according to the protocol described earlier [21]. The puppy then served as the donor animal/parasite bank for subsequent inoculation into the experimental animals.
Experimental animal inoculation: A total of ten dogs (mongrels) of both sex were randomly separated into two experimental groups (A \& B) of five dogs each. Group B were inoculated with $1 \mathrm{ml}$ of the inoculums containing $10^{4}$ parasitized erythrocytes per ml (1-4 parasites per microscopic field), while those in group A were left as uninfected (control).

On the day of challenge infection, and for a period of 14 days, the vital parameters (temperature, pulse and respiratory rates) were taken on alternate days. The dogs were observed daily for clinical signs. Special attention was given to behaviour, spleen size, size of lymph nodes and colour of the mucous membranes of mouth and eyelid as described previously [22]. Blood samples for biochemical analysis were collected daily from dogs in group B (infected) and compared with those in group A (uninfected). Plain sample bottles were used for biochemical analysis, fluoride bottles were used for glucose determination, while EDTA bottle was used for haematological analysis.

Biochemical parameters were determined using a biochemical auto-analyzer (Vital Scientific Microlab 300, Netherland). Analyses were also performed using the standard methods and original reagents of the manufacturers.

Following manifestation of clinical sign between day 2-14 and after the experiment at day 21; dogs in group B (infected) were treated with a single deep intramuscular injection of Diminazeneaceturate at a dose rate of $3.5 \mathrm{mg} / \mathrm{kg}$ body weight.

Statistical analysis: Data collected were subjected to one-way analysis of variance (ANOVA) and $\mathrm{p} \leq 0.05$ regarded as significant [23].

\section{Results}

Following infection, the major clinical signs seen were inappetence $(100 \%)$ two days post infection, followed by anorexia (40\%) and lethargy (100\%) on third day post infection. On the fourth day, the major clinical signs recorded in addition to the former ones were pallor of mucous membranes of the mouth and eyelid (100\%) and emaciation (100\%); on day five, 
Table-2. Mean \pm SD (range) of vital parameters of experimental dogs.

\begin{tabular}{lccc}
\hline Group & ${\text { Temperature }\left({ }^{\circ} \mathbf{C}\right)}$ & Pulse rate(beats/m) & Respiratory rate(breaths/m) \\
\hline A & $38.7 \pm 0.81^{\mathrm{a}}(38.1-39.4)$ & $76 \pm 15.00^{\mathrm{a}}(64-90)$ & $21 \pm 4.56^{\mathrm{a}}(12-34)$ \\
$\mathrm{B}$ & $39.4 \pm 1.4^{\mathrm{b}}(38.0-40.5)$ & $129 \pm 44.21^{\mathrm{b}}(50-196)$ & $31 \pm 12.76^{\mathrm{b}}(13-88)$ \\
\hline
\end{tabular}

$\mathrm{A}=$ Control, $\mathrm{B}=$ infected, $\mathrm{m}=$ minutes, $\mathrm{SD}=$ standard deviation. Column variables with different superscripts $(\mathrm{a} \& \mathrm{~b})$ are statistically significant $(p<0.05)$.

Table-3. Mean \pm SD (range) of serum enzymes and metabolites of experimental dogs.

\begin{tabular}{lcccc}
\hline Group & ALP $(\boldsymbol{\mu} / \mathbf{l})$ & ALAT $(\boldsymbol{\mu} / \mathbf{l})$ & Creatinine $(\boldsymbol{\mu m} / \mathbf{l})$ & Glucose $(\mathbf{m m o l} / \mathbf{l})$ \\
\hline $\mathrm{A}$ & $51.67 \pm 1.6^{\mathrm{a}}(14-91)$ & $8.27 \pm 2.0^{\mathrm{a}}(2-21)$ & $91.73 \pm 1.3^{\mathrm{a}}(65-108)$ & $5.35 \pm 2.1^{\mathrm{a}}(4.6-6.0)$ \\
$\mathrm{B}$ & $58.50 \pm 1.4^{\mathrm{b}}(37-149)$ & $15.70 \pm 1.8^{\mathrm{b}}(8-46)$ & $78.10 \pm 1.2^{\mathrm{b}}(68-100)$ & $2.86 \pm 2.4^{\mathrm{b}}(1.6-3.6)$ \\
\hline
\end{tabular}

ALP = Alkaline phosphatase, ALAT = Alanine amino transferase. Group A = Uninfected untreated (control), Group B = Infected group, $S D=$ Standard deviation. Column variables with different superscripts $(a \& b)$ are statistically significant $(p<0.05)$.

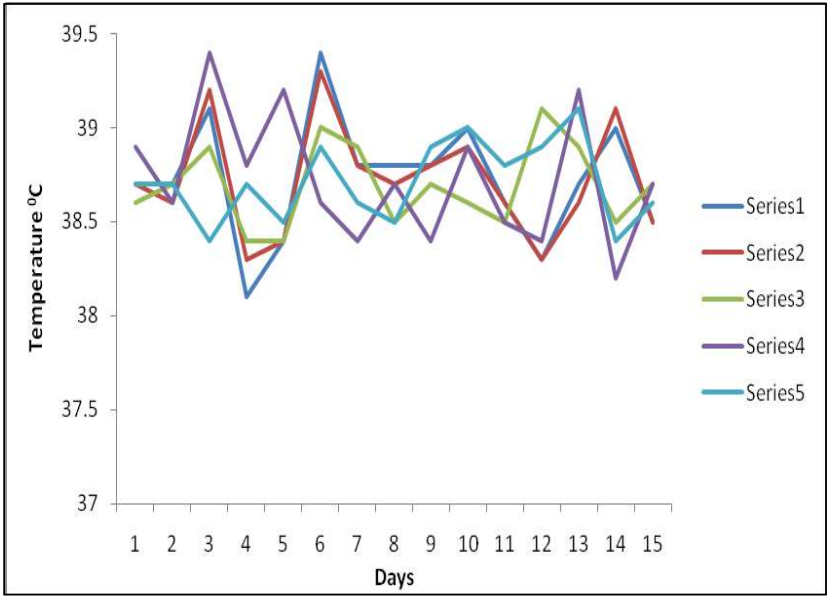

Figure-1a. Temperature of dogs in group A (control).

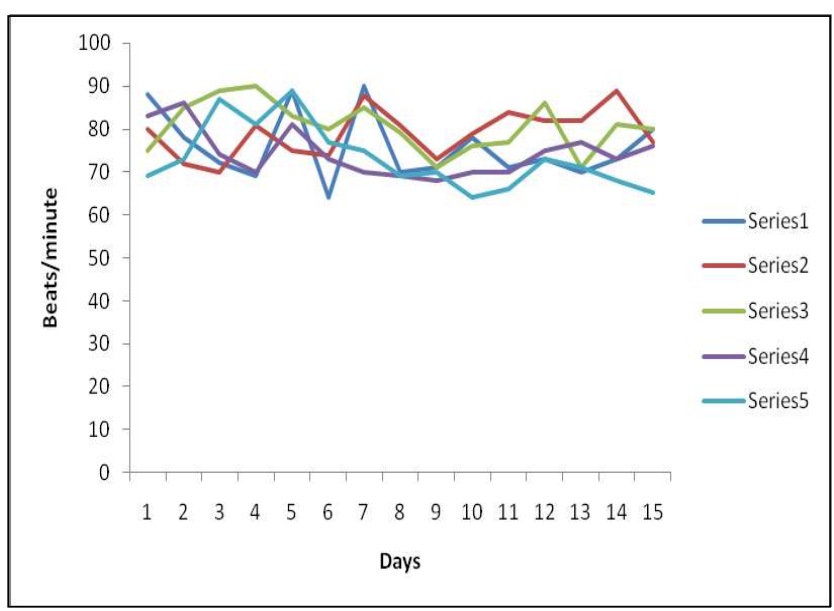

Figure-2a. Pulse rates of dogs in Group A (Control)

muscle tremor $(20 \%)$, respiratory distress $(10 \%)$; on day six, nervousness (20\%), drooling salivation (20\%) and haemoglobinuria $(80 \%)$; on day seven, mucoid ocular discharge (40\%); followed by death of one dog $(1 \%)$ at day eight post infection. Other clinical signs recorded between day 1-14 post infection included ascites, edematous swelling of the whole body and hair erection (Table-1).

There was a significant rise $(p<0.05)$ for all the vital parameters during the early course of infection compared with the control (Table-2). All infected dogs developed fever and it was first recorded two days post

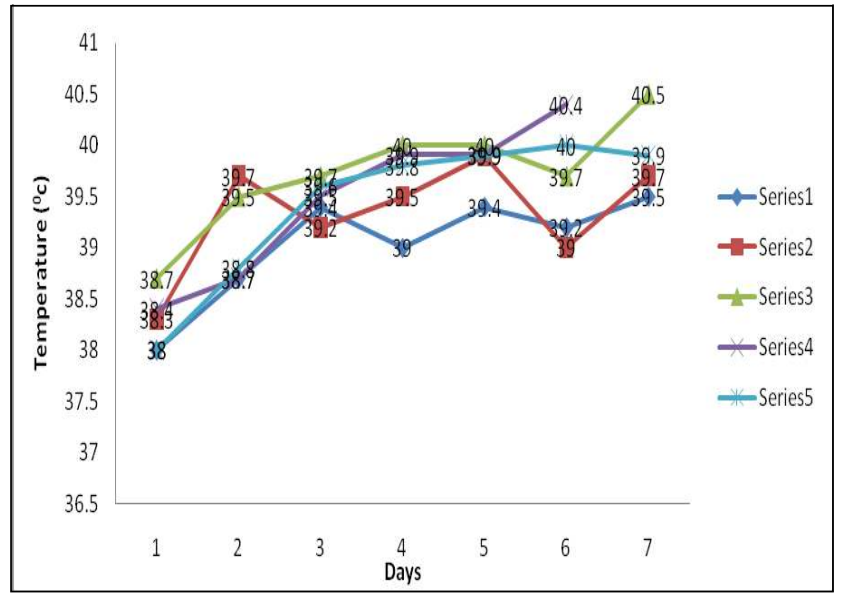

Figure-1b: Temperature responses of dogs in group B (infected). Each point on the horizontal axis represent 2 days.

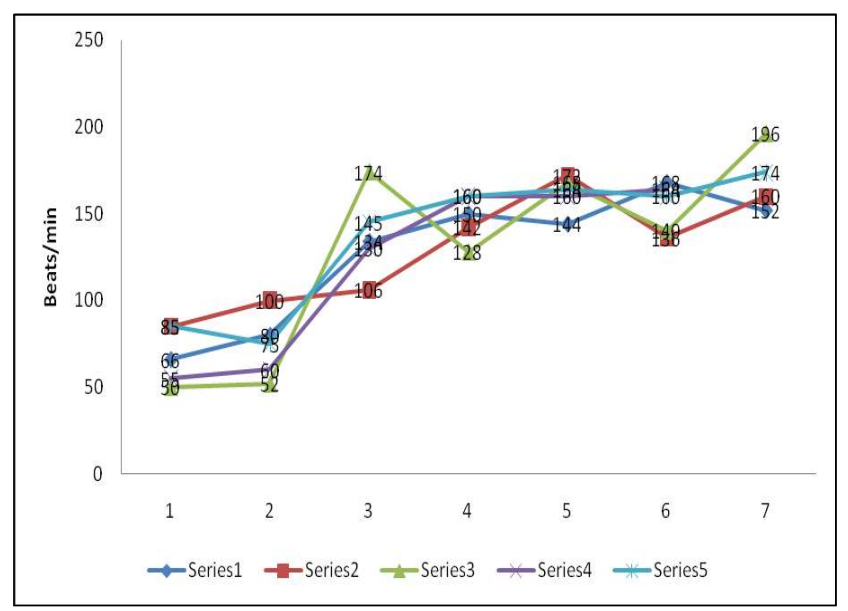

Figure-2b: Pulse rates of dogs in Group B (infected). Each point on the horizontal axis represent 2 days.

infection, reaching its peak between day 6 and 14 post infection (Fig.1b) as compared with the uninfected (control) group which showed with undulating pattern (Fig.1a). The body temperature of three dogs in group B (infected) was restored to normal values within 2-3 day post chemotherapeutic treatment, even though temperature in some dogs had started resolving prior to treatment. However one dog still showed a relapse/undulating fever post treatment.

There was a transient rise in the pulse reaching a peak of 196 per minute in 4 (80\%) dogs (Fig.2b); while $1 \mathrm{dog}(20 \%)$ recorded fall in pulse to as low as 27 per 


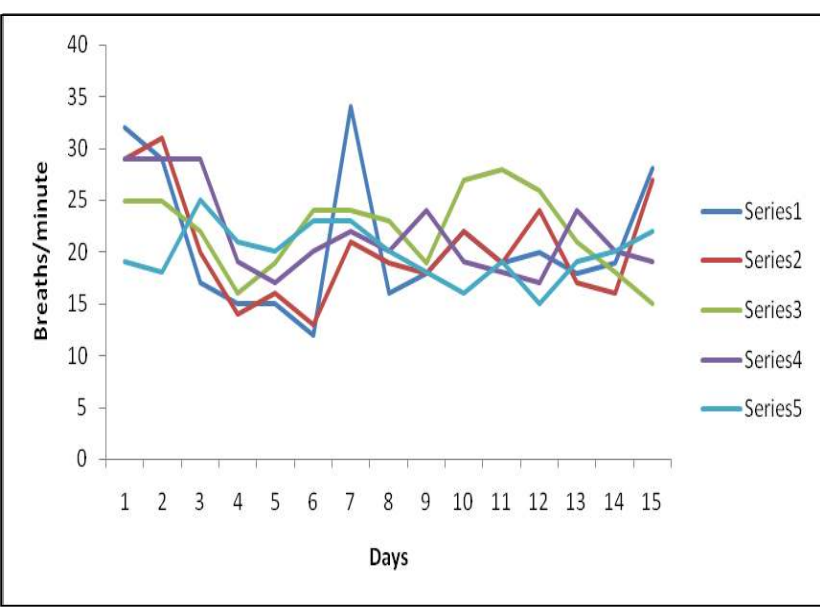

Figure-3a. Respiratory rates of dogs in Group A (Control)

minute; as compared with the uninfected (control) group that showed undulating patterns ranging from 64-90 beats per minute (fig.2a). Following Chemotherapeutic treatment, there was a sharp drop in pulse rate in dogs recording higher pulse to as low as 55 per minute then followed by a transient raise to normal value within 3-4 days.

Generally, there was a transient raise in the respiratory rate of all infected dogs reaching a peak of 90 breaths per minute (figure $3 b$ ) when compared with the uninfected (control) group that ranges between 1234 cycles per minutes (figure $3 \mathrm{a}$ ). But, following chemotherapeutic treatment an undulating pattern of breath has been recorded with an initial raise, then followed by a drop in most (90\%) of the dogs within 24 days post treatment, before restoring to normal.

The mean \pm standard deviation (range) values of serum enzymes and metabolites of the experimental dogs are shown in Table-3. There was a significant ( $\mathrm{p}<$ $0.05)$ rise in alkaline phosphatase (ALP) in infected dogs $(58.50 \pm 1.4)$ compared with the control group $(51.67 \pm 1.6)$. Also, there was a significant rise ( $\mathrm{p}<$ 0.05 ) in the alanine amino transferase (ALAT) values of infected group compared with the control values $(8.27 \pm 2.0)$. However, the mean \pm standard deviation values for creatinine of infected group $(78.10 \pm 1.2)$ was significantly lower $(\mathrm{p}<0.05)$ than that of the control $(91.73 \pm 1.3)$. Similarly, the glucose values for infected group $(3.80 \pm 2.3)$ were significantly $(\mathrm{p}<0.05)$ lower than that of control $(5.35 \pm 2.1)$.

\section{Discussion}

This study clearly shows that canine babesiosis associated with Babesia canis runs in two courses (acute and chronic) in West African dogs with the first clinical sign being recorded two days post infection, followed by death 8 days post infection, and others show chronic convalescent forms. The major clinical signs seen in this study were associated with intravascular haemolysis of the red blood cells in circulation, resulting in anaemia depicted as the pallor of mucous membrane of the mouth and eyelid. The sudden change

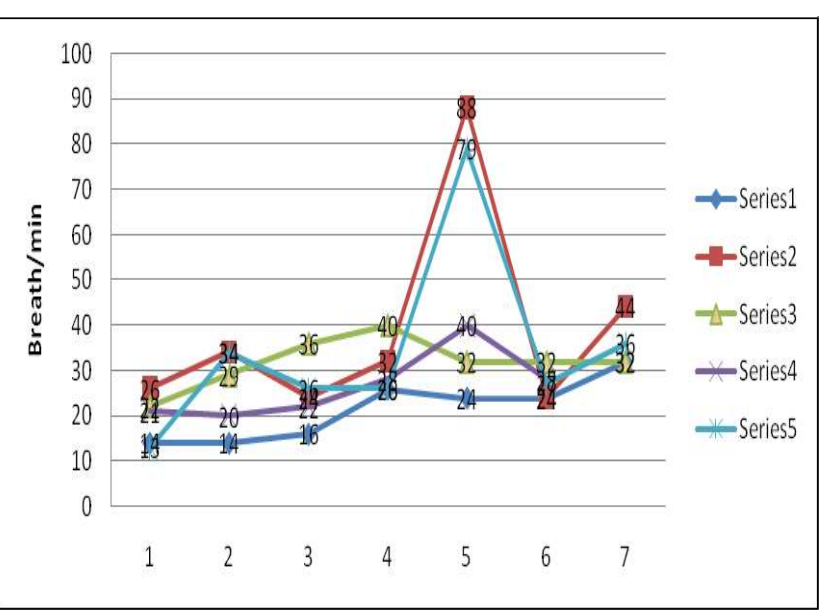

Figure-3b: Respiratory rates of dogs in Group B (infected). Each point on the horizontal axis represent 2 days.

in appetite and lethargy may be as a result of fever. The fever was as a result of the presence of parasites in the circulating blood as foreign bodies thereby triggering the release of vasoactive amines like cytokines.

Edematous body swelling caused by buildup and retention of abnormal amounts of fluid in the body may be due to hypoproteinaemia and hypoalbuminaemia caused by a decrease in plasma proteins and the lack of capillary colloidal osmotic pressure or the pressure needed to pull back the fluid into the vascular space. Usually, the plasma protein is what causes the fluid to be pulled back into the capillaries from the tissue spaces. So, when there is a decrease in plasma proteins as a result of an increased intravascular hemolysis of red blood cells, this pressure decreases and fluid starts to accumulate in the tissue spaces.

On the other hand, the liver cannot synthesize these plasma proteins without certain amino acids which can only be achieved by dietary intake. With anorexia and mal-absorption, there is insufficient intake of these amino acids resulting to malnutrition. Although many cases of malnutrition in dogs are connected to neglect or an insufficient diet, parasites may also create malnutrition in canines resulting to emaciation, which is the easiest sign of malnutrition as seen in this research.

Biochemical profile abnormalities in Babesia canis infection are related to both the severity of the disease and the degree of hypoxia as previously described [17,24]. In this study, the main biochemical changes were hypoproteinaemia, hypoalbuminaemia, increased serum activity of alkaline phosphatase and alanine amino transferase activity in majority of the infected dogs. This finding concurs with the previous reports [17-20,25]. Alkaline phosphatase (ALP) values in plasma were affected after challenge infection with a significant $(\mathrm{p}<0.05)$ rise in ALP values of infected dogs compared to the uninfected (control) group.

There was a clear trend of increasing values of ALP from 2-4 folds in most of the infected dogs (80\%) starting at day four post infection. While $20 \%$ of the dogs showed decrease in values below normal, though 
the difference is not much. This finding agrees with the report by [24] from Warsaw, Poland, who reported that the primary abnormalities found in affected dogs have increased serum activity of transaminases and alkaline phosphatase (ALP), suggesting that these abnormalities resulted from hepatopathy, renal failure and fasting. But, contradicts the report from Punjab (India) [26] and Bangkok (Thailand) [27] who evaluated hematological and biochemical changes in blood samples of some naturally infected dogs and reported that the biochemical values were within normal range but serum biochemistry varied for each dog. Even though, report from India was based on natural infection and that from Thailand was a retrospective study, the reason for such difference is not clear.

There was a decrease in serum activity of creatinine in most of the infected dogs as opposed to the report by [25] whose work was conducted under natural condition. The initial decrease in creatinine values may be as a result of dilution of blood. However, some few dogs showed an increase.

There was a general decrease in the blood glucose levels in most (90\%) of the infected dogs. That was as a result of sepsis causing anorexia, impaired hepatic function and increase in breakdown in the system. This finding was in consonant with the report by [28], who reported that the increased non-insulin mediated glucose consumption is believed to be induced by inflammatory mediators, more especially in macrophage-rich tissues like the spleen, liver and the lungs; at the same time regarded as a poor prognostic indicator. This condition has also been reported to cause muscle damage [29] when used creatine phosphokinase as indicator for muscle damage in some Babesia canis infected dogs. Hypoglycemia had also been reported to be one of the conditions that lead to high mortality in canine babesiosis [30].

\section{Conclusion}

It can be concluded that canine babesiosis associated with Babesia canis runs an acute course in naïve dogs in Nigeria, with few chronic forms and the incubation period usually varies 2-14 days, starting from 2 days after infection in acute cases to 7 days in chronic cases and lasting through 14 days. The disease is associated with a highly intravascular haemolytic anaemia which is usually regenerative and normocytic normochromic initially to microcytic hypochromic in the later stage of the disease. Diminazene aceturate at a dose rate of $0.3 \mathrm{mg} / \mathrm{kg}$ body weight coupled with hematinics and fluid therapy proves effective in clearing the system against parasitaemias and revitalizing the animal to normal within 2 weeks of giving the chemo-therapeutics.

\section{Authors' contributions}

MIA conceived and designed the experiment; MK performed the research and drafted the manuscript; $\mathrm{AAB}$ supervised the research and reviewed the manu- script; AWM revised the manuscript; JL analyzed the data. All authors read and approved the final manuscript.

\section{Acknowledgements}

The authors are grateful to Professor Idris Lawal of the Department of Veterinary Parasitology and Entomology, ABU, Zaria, for assistance in parasite isolation; Dr. O.O. Okubanjo for assisting in parasite identification, the entire staff of the Veterinary Teaching Hospital ABU Zaria and Protozoology laboratory, Department of Veterinary Parasitology and Entomology, Ahmadu University Zaria for providing all the necessary assistance during parasite isolation. The authors are also thankful to Management of Veterinary Teaching Hospital, University of Maiduguri and Parasitology Laboratory, Department of Veterinary Microbiology and Parasitology, Faculty of Veterinary Medicine, University of Maiduguri for their assistance during the course of the research and partial funding.

\section{Competing interests}

The authors declare that they have no competing interests.

\section{References}

1. Goo, Y., Jia, H. and Terkawi, M. (2009) Identification, localiszation and serological characterization of a Babesia gibsoni 22-kDa protein. Exp. Parasitol. 23: 273-6.

2. Di Cicco, M.F. and Birkenheuer, A.J. (2012) Canine babesiosis. Consultant on call/Clinician's brief 31-35.

3. Matsuu, A., Ono, S. and Ikadai, H. (2005) Development of a SYBR green real-time PCR assay for quantitative detection of B. gibsoni (Asia genotype) DNA. J. Vet. Diagn. Invest. 17: 569-73.

4. Schoeman, J.P. (2009) Canine babesiosis. Onderstepoort J Vet Res, 76: 59-66.

5. Cacciò, S.M., Antunovic, B., Moretti, A., Mangili, V., Marinculic, A., Rafaj Barc, R., Slemenda, S.B. and Pieniazek, N.J. (2002) Molecular characterisation of Babesia canis canis and Babesia canis vogeli from naturally infected European dogs, Vet. Parasitol. 106: 285-292.

6. Ayoob, A.L.; Hackner, S.G.; and Prittie, J. (2010) Clinical management of canine babesiosis. J. Vet. Emerg. Crit. Care 20(1), 77-89.

7. Matjila, P.T., Penzhorn, B.L., Bekker, C.P., Nijhof, A.M. and Jongejan, F. (2004) Confirmation of occurrence of Babesia canis vogeli in domestic dogs in South Africa. Vet. Parasitol.122: 119-125.

8. Jefferies, R., Ryan, U.M., Jardine, J., Broughton, D.K., Robertson, I.D. and Irwin, P.J. (2007) Blood, bull terriers and babesiosis. Further evidence for direct transmission of Babesia gibsoni in dogs. Aust. Vet. J. 85:459-460.

9. Bostrom, B., Wolf, C., Greene, C., and Peterson, D.S. (2008) Sequence conservation in the rRNA first internal transcribed spacer region of Babesiagibsoni genotype Asia isolates. Vet Parasitol., 152:152-157.

10. Fukumoto, S., Suzuki, H., Igarashi, I. and Xuan, X. (2005) Fatal experimental transplacental Babesia gibsoni infection in dogs. Int. J. Parasitol. 35: 1031-1035.

11. Ogo, N.I., Lawal, A.I., Okubanjo, O.O., Kamani, J. and Ajayi, O.O. (2011) Current status of canine babesiosis and the situation in Nigeria: A review. Niger. Vet. J. 32(2):69-78.

12. Carli, E., Tasca, S., Trotta, M., Furlanello, T., Caldin, M., Solano-Gallego, L. (2009) Detection of erythrocyte binding IgM and IgG by flow cytometry in sick dogs with Babesia canis canis or Babesia canis vogeli infection. Vet. Parasitol. 162: $51-57$. 
13. Rafaj, R.B., Matijatko, V., Kis, I., Kucer, N., Zivicnjak, T., Lemo, N., Zvorc, Z., Brkljacic, M. and Mrljak, V. (2009) Alterations in some blood coagulation parameters in naturally occurring cases of canine babesiosis. Acta Veterinaria Hungarica 57, 295-304.

14. Irwin, P.J. (2010) Canine babesiosis. Vet. Clin. Small Anim. 40:1141-1156.

15. Nel, M., Lobetti, R.G. and Keller, N. (2004) Prognostic value of blood lactate, blood glucose and hematocrit in canine babesiosis. J. Vet. Intern Med 18: 471-6.

16. Matijatko, V., Mrljak, V. and Kis, I. (2007) Evidence of an acute phage response in dogs naturally infected with Babesia canis. Vet. Parasitol. 144: 242-250.

17. Pagès, J.P., Vidor, E., Trouillet, J.L., Bissuel, G., Lecointre, O. and Moreau, Y. (1990) Description clinique, hématologique et sérologique de 133 cas de babésiose canine,Prat. Méd. Chir. Anim. Comp.25:89-97.

18. Bourdeau, P. and Guelfi, J.F. (1995) La babésiose canine à Babesia canis, Point Vét.27: 11-24.

19. Vercammen, F., De Deken, R. and Maes, L. (1997a) Haematological and biochemical profile in experimental canine 'babesiosis' (Babesia canis), Vlaams Diergenceskundig Tijdschrift.66: 174-178.

20. Lobetti, R.G. (2000) Canine babesiosis. In: M.J. Day, A. Mackin and J.D. Littlewood, BSAVA Manual of Canine and Feline Haematology and Transfusion Medicine, BSAVA, Gloucester. Pp. 85-91.

21. Schetters,Th. P.M, Kleuskens, J.A.G.M., Van De Crommet, J., De Leeuw, P.W.J., Finizio, A.L. and Gorenflot, A. (2009) Systemic inflammatory responses in dogs experimentally infected with Babesia canis; a haematological study. Vet. Parasitol. 162(1-2): 7-15.
22. Schetters, T.P. (1994) Vaccination against canine babesiosis. International forum on babesiosis. Nice, France. Pp. 19, 1994.

23. GraphPad Software Instat (2003) Guide to choosing and interpreting statistical tests. GraphPad software Inc. www.graphpad.com 5757 Oberlin Drive No.110 San Diego CA92121 USA(Instat Guide 153pp).

24. Zygner, W., Rapacka, G., Gojska-Zygner, O., Dlugosz, E. and Wedrychowicz, H. (2007) Biochemical abnormalities observed in serum of dogs infected with large Babesia in Warsaw (Poland). Pol J Vet Sci. 10 (4): 245-253.

25. Furlanello, T., Fiorio, F., Caldin, M., Lubas, G. and SolanoGallego, L. (2005) Clinicopathological findings in naturally occuring cases of babesiosis caused by large Babesia from dogs of northeastern Italy, Vet. Parasitol. 134:77-85.

26. Shah, S.A., Sood, N.K. and Tumati, S.R. (2011) Haematobiochemical changes in natural cases of canine babesiosis. Asian Journal of Animal Sciences. 5(6): 387-392.

27. Niwetpathomwat, A., Techangamsuwan, S.,Suvarnavibhaja, S., and Assarasakorn, S. (2006) A retrospective study of clinical haematology and biochemistry of canine babesiosis on hospital populations in Bangkok, Thailand. Comp Clin Pathol. 15(2): 110-112.

28. Amie, K. (2009) Hypoglycemia. Small Anim Crit. Care Med. 69: 295-299.

29. Jacobson, L.S. and Lobetti, R.G. (1996) Rhabdomyolysis as a complication of canine babesiosis. J. Small Anim. Pract. 37:286-291.

30. Keller, N., Jacobson, L.S., Nel, M., de Clerq, M., Thompson, P.N. and Schoeman, J.P. (2004) Prevalence and risk factors of hypoglycemia in virulent canine babesiosis. J. Vet. Intern. Med. 18:265-270. 